\title{
Observing how others lift light or heavy objects: time-dependent encoding of grip force in the primary motor cortex
}

\author{
Kaat Alaerts • Toon T. de Beukelaar • \\ Stephan P. Swinnen · Nicole Wenderoth
}

Received: 25 June 2011 / Accepted: 5 September 2011/Published online: 20 September 2011

(C) Springer-Verlag 2011

\begin{abstract}
During movement observation, corticomotor excitability of the observer's primary motor cortex (M1) is modulated according to the force requirements of the observed action. Here, we explored the time course of observation-induced force encoding. Force-related changes in M1-excitability were assessed by delivering transcranial magnetic stimulations at distinct temporal phases of an observed reach-grasp-lift action. Temporal changes in force-related electromyographic activity were also assessed during active movement execution. In observation conditions in which a heavy object was lifted, M1-excitability was higher compared to conditions in which a light object was lifted. Both during observation and execution, differential force encoding tended to gradually increase from the grasping phase until the late lift phase. Surprisingly, however, during observation, force encoding was already present at the early reach phase: a time point at which no visual cues on the object's weight were available to the observer. As the observer was aware that the same weight condition was presented repeatedly, this finding may indicate that prior predictions concerning the upcoming weight condition are reflected by M1 excitability. Overall, findings may provide indications that the observer's motor
\end{abstract}

Electronic supplementary material The online version of this article (doi:10.1007/s00426-011-0380-1) contains supplementary material, which is available to authorized users.

K. Alaerts $(\varangle) \cdot$ T. T. de Beukelaar · S. P. Swinnen ·

N. Wenderoth

Motor Control Laboratory, Research Centre of Movement

Control and Neuroplasticity, Department of Biomedical

Kinesiology, Group Biomedical Sciences,

Katholieke Universiteit Leuven, Tervuursevest 101,

3001 Heverlee, Belgium

e-mail: Kaat.Alaerts@faber.kuleuven.be system represents motor predictions as well as muscular requirements to infer the observed movement goal.

\section{Introduction}

In social interactions, humans demonstrate a remarkable ability to understand and interpret the behavior of others in a seemingly effortless way. With regard to this topic, neuroscience and social cognition research have increasingly focused on the role of the so-called mirror neuron system (MNS), which is believed to mediate people's ability to understand or 'read' the actions made by others (Gallese, Keysers, \& Rizzolatti, 2004; Gallese, 2009; Sinigaglia \& Sparaci, 2010). Single neuron recordings in macaque monkeys first demonstrated the existence of mirror neurons in ventral premotor (Di Pellegrino, Fadiga, Fogassi, Gallese, \& Rizzolatti, 1992; Rizzolatti, Fadiga, Gallese, \& Fogassi, 1996) and inferior parietal cortex (Fogassi et al., 2005), which fire when the monkey performs a specific action, and also when it observes the same action performed by another individual. Using movement observation paradigms, functional imaging studies demonstrated the existence of a similar fronto-parietal mirror neuron network in humans (Buccino et al., 2001; Grafton, Arbib, Fadiga, \& Rizzolatti, 1996; Grezes, Armony, Rowe, \& Passingham, 2003). Based on its remarkable properties, the human MNS is hypothesized to act as a neural mapping mechanism between the observation and execution of bodily movements, which enables observers to 'simulate' or 'resonate with' perceived movements using his/her own motor system (Gallese, 2009; Rizzolatti \& Sinigaglia, 2010).

Among other experimental approaches, transcranial magnetic stimulation (TMS) has been a widely adopted 
technique to study the human MNS at work, as it provides fast and focal measurements of corticomotor excitability within the primary motor cortex (M1) of subjects observing actions performed by others. Using TMS, a number of studies have shown that M1 becomes facilitated during the mere observation of actions (Fadiga, Fogassi, Pavesi, \& Rizzolatti, 1995; Strafella \& Paus, 2000), and, moreover, that these modulations in M1 excitability are highly muscle specific (Alaerts, Swinnen, \& Wenderoth, 2009a; Alaerts, Heremans, Swinnen, \& Wenderoth, 2009b; Maeda, Kleiner-Fisman, Pascual-Leone, 2002; Romani, Cesari, Urgesi, Facchini \& Aglioti, 2005; Urgesi, Candidi, Fabbro, Romani \& Aglioti, 2006) and synchronized to the temporal dynamics of observed movements (Borroni, Montagna, Cerri, \& Baldissera, 2005; Gangitano, Mottaghy, \& Pascual-Leone, 2001; Montagna, Cerri, Borroni, \& Baldissera, 2005). For example, Montagna et al. (2005) recorded TMS-induced motor evoked potentials (MEPs) from hand and finger muscles at different time points of an observed reaching and grasping action. Also, the EMG activity of these muscles was recorded while the subjects were actually performing a movement similar to the one observed. In all subjects and for each muscle, the authors found a clearcut correspondence between the time course of the excitability modulation of MEPs and the temporal pattern of EMG recruitment, suggesting that 'motor resonance' subliminally activated the same motor pathways that would be overtly recruited when actually performing the observed movement.

In addition to the temporal dynamics of observed actions, previous studies also demonstrated that the muscular force requirements of an observed action are represented in the observer's M1 (Alaerts et al., 2010a; Alaerts, Swinnen, \& Wenderoth, 2010b). In these experiments, corticomotor excitability was measured in the hand area of M1, while subjects observed the lifting of objects with different weights. The type of action 'grasping and lifting the object' was always identical, but the grip force varied according to the object's weight. Results indicated that the muscle-specific facilitation pattern of M1 modulated in accordance with the force requirements of the observed actions, such that corticomotor excitability was considerably higher when observing heavy object lifting compared to light object lifting (Alaerts et al., 2010a). Interestingly, in a series of follow-up experiments, it was shown that weight/force-related excitability in the observer's M1 persists when only kinematical motion trajectories-associated with lifting heavy or light objects-were presented to the observers (Alaerts et al., 2010b). Moreover, a strong preference for using kinematic features was shown to exist when kinematic cues and intrinsic object properties provided distinct information on the force requirements of an observed lifting action, i.e., observing the kinematic trajectory associated with lifting heavy or light objects substantially influenced the measured corticomotor excitability within M1, whereas the effects of intrinsic object characteristics of the lifted object (i.e., its filling degree) were negligible (Alaerts et al., 2010b).

However, in these previous experiments, weight/forcedependent modulations in corticomotor excitability were only assessed during the late lifting phase, a time point at which most of the kinematical motion trajectory of the observed movement had already unfolded. Considering previous findings of time course-dependent M1 modulations during movement observation (Borroni et al., 2005; Gangitano et al., 2001), the present study aimed to investigate the extent of force encoding in M1 at various time points of the movement trajectory. Video clips were presented displaying the right hand of an actor reaching for an object, grasping it with a whole handgrip and lifting it to place it on an elevated platform (reach-grasp-lift-place). The manipulated objects were identical in appearance, but differed in weight (heavy or light). Single-pulse TMS stimulations were delivered during (i) reach, (ii) early grasp, (iii) late grasp/initial lift, (iv) late lift, (v) place and (vi) return of the hand to its initial position. Motor evoked potentials were recorded from the opponens pollicis (OP) thumb muscle. Importantly, information on the lifted weight and, correspondingly, the produced force was only evident from differences in the kinematic trajectory of the movement.

Overall, we hypothesized weight-dependent M1 facilitation to evolve predominantly during the late grasp and/or lifting phases, and to be minimal at the reach, early grasp and return phase. Moreover, if weight encoding in M1 is tightly time locked to the observed kinematical movement trajectory, we hypothesize weight-dependent differences in M1 facilitation to gradually increase the more kinematical cues on object weight accumulated by the observer.

\section{Materials and methods}

Experiment 1: measurements of corticomotor excitability during the observation of light or heavy object lifting

\section{Participants}

Sixteen subjects participated in the study $(8$ males, 8 females, mean age 23, SD 4.4 years). All participants were right-handed, as assessed with the Edinburgh Handedness Questionnaire (Oldfield, 1971) and were naive about the purpose of the experiment. Written informed consent was obtained before the experiment and all subjects were screened for potential risk of adverse effects during TMS. 
The experimental procedure was approved by the local Ethics Committee for Biomedical Research at the Katholieke Universiteit Leuven and conformed with The Code of Ethics of the World Medical Association (Declaration of Helsinki).

\section{Electromyographic recordings and TMS}

Surface electromyography (EMG) was performed with $\mathrm{Ag}-$ $\mathrm{AgCl}$ electrodes (Blue Sensor SP) placed over the muscle belly and aligned with the longitudinal axis of the muscle. EMG activity was recorded from the right opponens pollicis (OP) thumb muscle. Focal TMS was performed by means of a 70-mm figure-of-eight coil connected to a Magstim 200 stimulator (Magstim, Whitland, Dyfed, UK). The coil was positioned over the left hemisphere, tangentially to the scalp with the handle pointing backward and laterally at $45^{\circ}$ away from the mid-sagittal line, such that the induced current flow was in a posterior-anterior direction, i.e., approximately perpendicular to the central sulcus. The optimal scalp position was defined as the position from which motor evoked potentials (MEPs) with maximal amplitude were recorded in the right OP muscle. The rest motor threshold (rMT) was defined as the lowest stimulus intensity evoking MEPs in the OP with an amplitude of at least $50 \mu \mathrm{V}$ in five out of ten consecutive stimuli (Rossini et al., 1994). Subjects' rest motor thresholds, expressed as a percentage of the maximum stimulator output, varied from 34 to $56 \%$ (mean $43 \%$ ). For all experimental trials, stimulation intensity was set at $130 \%$ of the subjects' rMT. EMG recordings were sampled at 5,000 Hz, (CED Power 1401, Cambridge Electronic Design, UK) amplified, band-pass filtered $(30-1,500 \mathrm{~Hz})$ and stored on a PC for off-line analysis. Signal Software (2.02 Version, Cambridge Electronic Design, UK) was used for TMS triggering and EMG recordings.

\section{General procedure}

Participants were seated in a comfortable chair in front of a Dell 1707 monitor (resolution, $1152 \times 870$ pixels; refresh frequency $60 \mathrm{~Hz}$ ) on which video clips [audio-video interleaved (AVI)] were displayed with a frame rate of $25 \mathrm{~Hz}$. Before the experiment, video clips were presented to the subjects to familiarize them with the experimental stimuli. Importantly, however, no explicit information was provided on the type of video presented (i.e., the observing subjects had no prior knowledge of the weight of the lifted objects in the videos). During the session, they were instructed to keep their hands and forearms as relaxed as possible and to pay full attention to the video presented. Vision of their own hand and forearm was never allowed. Muscle relaxation was monitored and, whenever EMG activity became apparent during data collection, the trial was discarded and repeated.

\section{Stimuli}

Stimuli consisted of two video clips presenting grasping and lifting of objects with identical appearance, but with different weights, respectively, $0.1 \mathrm{~kg}$ (light object) and $2.1 \mathrm{~kg}$ (heavy object). In both clips, the right hand of an actor reached for the object, grasped it with a whole handgrip and lifted it to place it on an elevated platform (reach-grasp-lift-place) (Fig. 1b). To conceal any muscleor skin-related cues concerning the exerted force, the actor's hand was covered with a glove and sleeve. Thus, the only visible difference between the two conditions was the kinematic profile of the trajectories shown in the video. MTrackJ software (Biomedical Imaging Group, Rotterdam, The Netherlands) was used to quantify the kinematic motion trajectories of the lifted objects as shown in the two video clips on a frame-by-frame basis. MTrackJ is an
Fig. 1 a Kinematic trajectory for lifting heavy (black line) or light (gray line) objects. TMS stimulations were delivered at six time points, corresponding to distinct temporal phases of the kinematical movement trajectory, namely during reach, early grasp, late grasp/initial lift, late lift, place and return. b Illustration of the sequence of events during observation of the reach-grasp-lift action. Video clips displayed the right hand of an actor reaching for an object, grasping it with a whole handgrip and lifting it to place it on an elevated platform

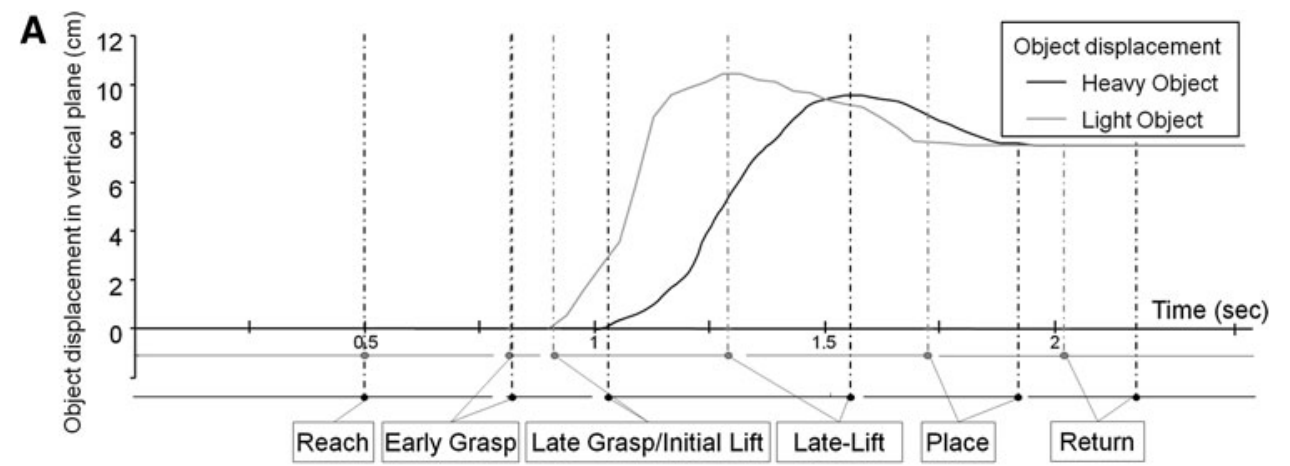

B

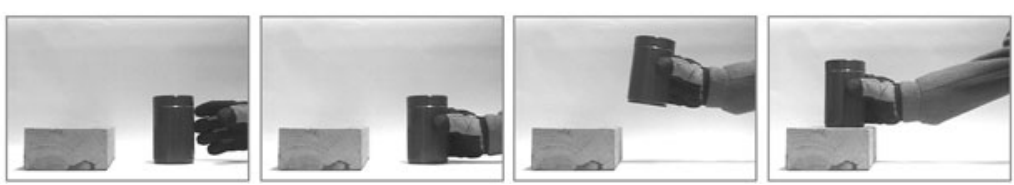


ImageJ plugin (National Institutes of Health, MD, USA) designed for manual tracking of moving objects in video sequences. The kinematic motion trajectory can be divided into four distinct phases, namely, the reach, grasp, lift and the place phase (Fig. 1a). Reach was defined as the time from the start of the movement until the point of handobject contact. Grasp lasted from the end of reach until the start of object displacement. Lift lasted from the start of object motion to the object's peak height and place from the peak height until the end of object displacement as the object was put on the shelf. The actor in the video had no prior knowledge of the weight of the to-be-lifted object, such that spontaneous 'typical' lifting actions were obtained. Overall, Fig. 1 shows that the grasp and lift duration differed considerably between heavy and light object lifting, whereas differences in duration were small for the reach and place phase. Vertical velocity was calculated from the position data derived from the frame-byframe analysis of the video and smoothed by a five-point sliding average. The averaged vertical velocity, calculated for the successive frames of the lifting phase, was, respectively, 0.021 and $0.014 \mathrm{~cm} / \mathrm{ms}$ for light and heavy object lifting.

Each of the two video clips was presented in blocks of six repetitions, i.e., the same video clip was shown six times in a row and subjects were aware of this blocked order. Within each block, single TMS pulses were delivered at six distinct time points of the movement sequence (one stimulation/video clip). Figure 1a shows the occurrence of TMS stimulation relative to the kinematic motion trajectory of the presented motion: (1) during the reach phase, stimulation occurred when the index finger was $4 \mathrm{~cm}$ before the object; (2) the early grasp point was defined at the point of hand-object contact; (3) the late grasp/initial lift point occurred just before the object was lifted from the table; (4) the late lift point occurred at the object's peak height; (5) the place point occurred at the end of object displacement, i.e., when the object was put on the shelf and the grip was released; and (6) the return point occurred $0.3 \mathrm{~s}$ after the place point when the hand moved away from the object. For each video, subjects watched 20 blocks resulting in 240 TMS pulses in total [i.e., 20 stimulations per video (2) per time point (6)]. Signal Software (2.02 Version, Cambridge Electronic Design, UK) was used to synchronize video presentation and TMS triggering. To insure that participants were attending the presented videos, they were instructed to judge the weight of the lifted object (light or heavy) after each block.

\section{Data reduction and analysis}

From the EMG data, peak-to-peak amplitudes of the MEPs were determined. Since EMG background activation is known to modulate the MEP amplitude (Devanne, Lavoie, \& Capaday, 1997; Hess, Mills, \& Murray, 1987), prestimulation EMG was assessed by computing root meansquare scores (RMS) across a 50-ms interval prior to the TMS stimulation. For each subject, mean and standard deviations of the EMG background scores were computed over all trials. Trials for which EMG background was above the mean +2.5 standard deviation were removed from the analysis. Trials for which the MEP amplitude was inferior to the mean EMG background were also discarded. Finally, extreme peak-to-peak amplitude values were considered as outliers and removed from the analysis when they exceeded $Q 3 \pm 1.5 \times(Q 3-Q 1)$, with $Q 1$ the first quartile and $Q 3$ the third quartile computed over the whole set of trials for each subject (Electronic Statistics Textbook, 2007, StatSoft, Inc. Tulsa). Following these three criteria, only $4.32 \%$ of all trials (of all subjects) were discarded from the analyses. To reduce between-subject variability in MEP size while maintaining between condition differences, MEPs of each individual were z-transformed using the mean and standard deviation of the MEP amplitudes measured over all trials and time points. Additionally, the MEP ratio was obtained by dividing the MEP amplitudes recorded during the heavy object conditions by MEP amplitudes recorded during the light object conditions ( $\left.\mathrm{MEP}_{\text {heavy }} / \mathrm{MEP}_{\text {light }}\right)$.

To analyze modulations in background EMG across observation conditions, RMS scores were also z-transformed and RMS ratios were calculated.

\section{Statistics}

MEP ratios To specifically explore weight/force-dependent M1 facilitation at distinct time points, one sample $\mathrm{T}$ tests were conducted on the MEP ratios to assess significant increases in MEP amplitudes for the 'heavy' over the 'light' observation condition [separately for all stimulation points (reach-early grasp-late grasp/initial lift-late liftplace-return)]. A ratio significantly larger than 1 indicates a facilitatory effect of observing how a heavy versus a light object is grasped, lifted and placed.

MEP z-scores $\quad \mathrm{Z}$-transformed MEP scores were subjected to a $2 \times 6$ repeated-measures analysis of variance (ANOVA) with the within factors 'weight' (light-heavy) and 'time point' (reach-early grasp-late grasp/initial liftlate lift-place-return). Significant effects were further analyzed using Fisher LSD post hoc tests (Statistica 7.0, StatSoft. Tulsa, USA).

Background EMG Similar statistical analyses were applied to the background EMG data (RMS ratios/RMS 
scores) to assess whether the MEP data were confounded by modulations in background EMG.

Experiment 2: measurements of muscle activity during the execution of light or heavy object lifting

\section{Participants}

Four new subjects ( 1 male, 3 females, mean age 21, SD 0.5 years) participated in an additional experiment to record the corresponding muscle activity during the actual execution of the whole hand reach-grasp-lift actions of a heavy or light object. All participants were right-handed, as assessed by the Edinburgh Handedness Questionnaire (Oldfield, 1971) and written informed consent was obtained before the experiment.

\section{General procedure}

Active markers (infrared-emitting diodes) were placed on the heavy/light object and on the nails of the thumb and index finger of the subjects to register the kinematic motion trajectories of the reach-grasp-lift actions by a threedimensional tracking system (at $100 \mathrm{~Hz}$ ) (Optotrak 3020, NDI, ON, Canada). During execution, EMG was simultaneously recorded from the right OP thumb muscle. Each subject performed the lifting of the heavy and light object ten times in a random order. Subjects had no prior knowledge on the weight of the to-be-lifted object, such that spontaneous 'typical' lifting actions were obtained.

\section{Data reduction and analysis}

Data were analyzed off-line to determine the distinct phases from the kinematics. Grip aperture was calculated as the euclidean distance between the markers on index finger and thumb. The grasp phase was defined as the time point when the fingers made contact with the object. This was determined based on the first derivative of the hand aperture (Vap), which was smoothed by a second-order Butterworth low-pass filter with a cutoff frequency of $20 \mathrm{~Hz}$. Point of contact was operationally defined as the time when the Vap fell below $20 \mathrm{~mm} / \mathrm{s}$. The start of the lift phase corresponded to the time point when the velocity of the vertical displacement measured by the object marker exceeded $20 \mathrm{~mm} / \mathrm{s}$. The lift phase ended when the highest point of the vertical displacement was reached. Finally, the place phase lasted from the time point of maximal vertical displacement until the hand released the object after it had been put down on the shelf. The moment of release was identified as the time point when Vap exceeded a velocity of $20 \mathrm{~mm} / \mathrm{s}$. Root mean-square scores were recorded from the EMG data within a 50-ms interval, which was centered around the six distinct time points describing (1) the reach point, occurring $0.3 \mathrm{~s}$ before hand-object contact; (2) the early grasp point, occurring at the beginning of the grasp phase (i.e., point of object contact); (3) the late grasp/initial lift point, occurring at the beginning of the lift phase (i.e., when the object was lifted from the table); (4) the late lift point, occurring at the highest point of the vertical object displacement; (5) the place point, occurring at the end of the place phase (i.e., when the hand just released the object); and (6) the return point, occurring $0.3 \mathrm{~s}$ after object release. In some additional trials, the EMG was recorded during maximal voluntary contraction (MVC) of the OP muscle.

EMG changes were normalized to subjects' musclespecific MVC scores, and EMG ratios were obtained by dividing the 'heavy object' EMG scores by the 'light object' EMG scores (EMG $\left.\mathrm{Emeavy}_{\text {h }} / \mathrm{EMG}_{\text {light }}\right)$.

\section{Statistics}

EMG ratios For all time points (reach-early grasp-late grasp/initial lift-late lift-place-return), one sample $T$ tests were conducted on the EMG ratios to assess significant increases in muscle activity for the 'heavy' over the 'light' condition.

EMG scores MVC-normalized EMG scores were subjected to a $2 \times 6$ repeated-measures analysis of variance (ANOVA) with the within factors 'weight' (light-heavy) and 'time point' (reach-early grasp-late grasp/initial liftlate lift-place-return). Significant effects were analyzed using Fisher LSD post hoc tests (Statistica 7.0, StatSoft. Tulsa, USA).

\section{Results}

During movement observation, all subjects attended to the stimuli and were able to report the weight of the lifted object after each block $(98.1 \%$ correct answers on average).

Experiment 1: MEP facilitation during observation of light or heavy object lifting

\section{MEP ratios}

As shown in Fig. 2a, MEP ratios $\left(\mathrm{MEP}_{\text {heavy }} / \mathrm{MEP}_{\text {light }}\right)$ were significantly above 1 for the reach point and the late lift point [reach: $t(15)=3.45 ; p<0.01$; late lift: $t(15)=2.38 ; p<0.05]$. As such, only at these two time points, MEP facilitation was significantly higher for the heavy object condition compared to the light object 

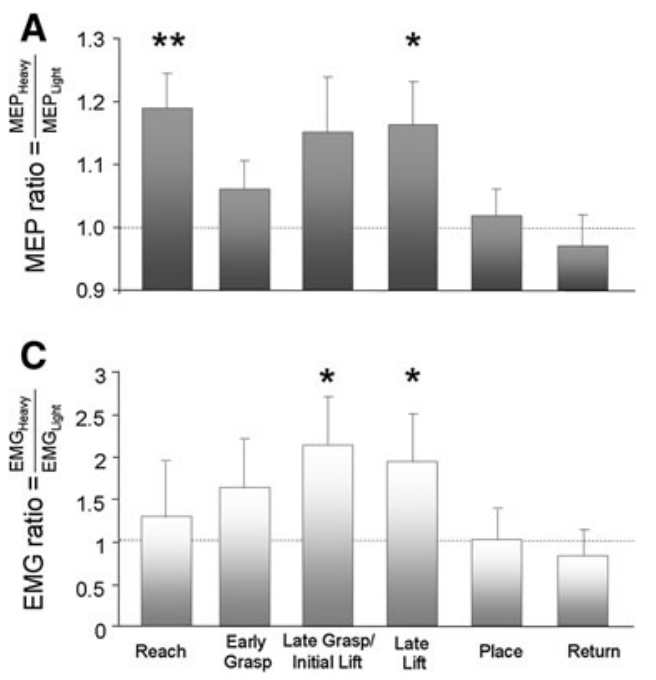

Fig. 2 Corticomotor excitability during movement observation at six time points ( $x$-axis) of the reach-grasp-lift action. a The MEP ratio ( $y$-axis) was obtained by dividing MEPs recorded when observing heavy weight conditions by MEPs recorded when observing light weight conditions $\left(\mathrm{MEP}_{\text {heavy }} / \mathrm{MEP}_{\text {light }}\right)$. A ratio significantly above 1 indicates a facilitatory effect of observing a heavy versus a light object $\left({ }^{*} p<0.05 ;{ }^{* *} p<0.01\right)$. Vertical lines denote standard error. b Group averaged MEP z-scores recorded during movement observation at six time points of the reach-grasp-lift action. For the heavy object condition, asterisks denote time points at which MEP z-scores were significantly higher $(p<0.05)$ compared to other indicated time points. For the light object condition, hash denote time points at which MEP z-scores were significantly higher $(p<0.05)$ compared to other indicated time points. $\mathbf{c}$ and $\mathbf{d}$ display EMG activity recorded

condition. For the late grasp/initial lift point, MEP ratio was also above 1 , but statistics revealed only a slight tendency toward significance due to large inter-subject variability $[t(15)=1.73 ; p=0.10]$.

\section{MEP z-scores}

The two (heavy versus light) by six (time points) repeatedmeasures ANOVA on the z-transformed MEP scores confirmed that weight-dependent MEP facilitation was modulated across time, as shown by a significant 'weight' $\times$ 'time point' interaction effect $[F(5,75)=2.5$; $p<0.05]$. Also, the main effect of 'weight' reached significance $[F(1,15)=4.6 ; p<0.05]$. Figure $2 \mathrm{~b}$ reports all z-normalized MEP scores separately for the heavy and light conditions.

Post hoc analysis of the interaction effect was conducted to explore the time courses of MEP modulation separately for the heavy and light observation condition (Fig. 2b).

For the heavy observation conditions, MEP facilitation was most pronounced at the reach and late lift point as differences in MEP facilitation only occurred at these points. MEP facilitation at the reach point was significantly

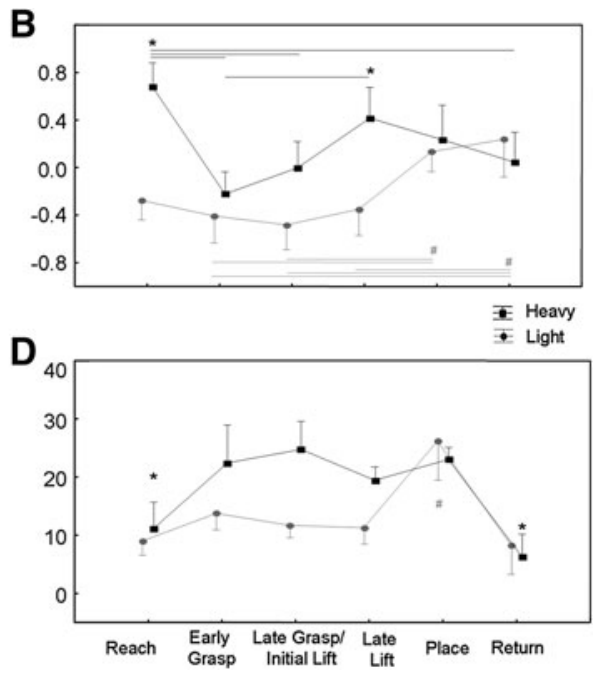

during active execution at six time points ( $x$-axis) of the reach-grasplift action. $\mathbf{c}$ The EMG ratio ( $y$-axis) was obtained by dividing EMG recorded when manipulating heavy weight objects by EMG recorded when manipulating light weight objects $\left(\mathrm{EMG}_{\text {heavy }} / \mathrm{EMG}_{\text {light }}\right)$. A ratio significantly above 1 indicates a facilitatory effect of manipulating a heavy versus a light object $(* p<0.05)$. Vertical lines denote \pm standard error. d Group averaged EMG scores recorded during movement execution at six time points of the reach-grasp-lift action. For the heavy object condition, asterisks denote time points at which EMG scores were significantly lower $(p<0.05)$ compared to all other time points. For the light object condition, hash denote time points at which EMG scores were significantly higher $(p<0.01)$ compared to all other time points

larger than MEP facilitation recorded at the early grasp, late grasp/initial lift and return point (all $p<0.05$ ). For the late lift point, only the comparison with the early grasp point reached significance $(p<0.05)$.

Surprisingly, for the light observation conditions, MEP facilitation was most pronounced at the place and return point as differences in MEP facilitation only occurred at these points. MEP facilitation at the place point was significantly larger compared to MEP facilitation at the early grasp and late grasp/initial lift point (both $p<0.05$ ). Similarly, at the return point, MEP facilitation was larger compared to the early grasp, late grasp/initial lift and late lift point (all $p<0.05)$.

\section{Background EMG}

Recorded MEP data were not confounded by modulations in background EMG, since RMS scores were generally small and condition-specific modulations were minimal. This was tested by conducting similar statistical analyses on the background EMG data. For all TMS stimulation points, RMS ratios were not significantly larger than 1 [all $t(15)<0.75 ; p>1$ ], and no significant 'weight' $\times$ 'time 
point' interaction effect was revealed from the ANOVA analysis on the $\mathrm{z}$-normalized RMS scores $[F(5,75)=1.14$; $p=0.34]$.

Experiment 2: EMG scores during execution of light or heavy object lifting

\section{EMG ratios}

As shown in Fig. 2C, EMG ratios $\left(\mathrm{EMG}_{\text {heavy }} / \mathrm{EMG}_{\text {light }}\right)$ were significantly above 1 for the late grasp/initial lift point $[t(3)=4.12 ; p<0.05]$ and late lift point $[t(3)=3.2$; $p<0.05]$. As such, only at these two time points, EMG activity was higher for the heavy object condition compared to the light object condition.

\section{EMG scores}

The repeated-measures ANOVA on the z-transformed EMG scores confirmed that weight-dependent EMG activity was modulated across time, as shown by a significant 'weight' $\times$ 'time point' interaction effect $[F(5$, $15)=3.91 ; p<0.05]$. Also the main effect of 'time point' reached significance $[F(5,15)=3.99 ; p<0.05]$. Figure $2 \mathrm{~d}$ reports all $\mathrm{z}$-transformed EMG scores separately for the heavy and light conditions.

Post hoc analysis of the interaction effect was conducted to explore the time courses of MEP modulation separately for the heavy and light conditions (Fig. 2d).

For the heavy condition, EMG activity was minimal at the reach and return point; as at these points EMG activity was significantly smaller compared to all other time points (all $\mathrm{p}<0.05$ ).

For the light conditions, on the other hand, a substantial peak in EMG activity was revealed only for the place point, at which EMG activity was significantly larger compared to all other time points (all $p<0.01$ ).

\section{Discussion}

The present study aimed to explore the time course of weight/force-dependent M1 facilitation during the observation of heavy versus light object lifting. TMS stimulations were delivered at distinct temporal phases of the kinematical movement trajectory, namely during (i) reach, (ii) early grasp, (iii) late grasp/initial lift, (iv) late lift, (v) place and (vi) return. Temporal changes in force-related EMG activity were also assessed during active action execution. Both during observation and execution, force encoding tended to gradually increase from the grasping until the late lifting phase. However, only during observation, TMS data consistently revealed an early peak in force-related M1 modulation already at the reach phase. No such force scaling was observed, however, during actual execution.

Time course-dependent force encoding in M1

During movement observation, M1 encoded the force requirements of observed movements. Considering distinct time points of grasping-lifting-placing action, it appeared that differences in M1 facilitation for observing heavy or light object manipulation were most pronounced during the late lifting phase, which is in agreement with previous findings (Alaerts et al., 2010a, 2010b). Closer inspection revealed that temporal variations in observation-induced M1 facilitation were most pronounced for the heavy object observation condition: in this condition, a gradual increase in M1 facilitation emerged during grasping and initial lifting, which peaked at the late lifting phase. For the light object condition, on the other hand, M1 facilitation remained relatively constant during grasping and lifting to peak substantially at the place phase. Even though this seems to be counter-intuitive at the first glance, the M1 facilitation pattern during movement observation mimics the temporal modulation in thumb muscle activity during actual execution: i.e., for manipulating the light object, muscle activity also peaked at the placing phase, whereas for heavy object manipulation, increases in muscle activity also emerged during the grasping and lifting phase. It appears that the acting subjects applied similarly high muscle forces to prevent objects from slipping during the placing of both heavy and light objects and, strikingly, these applied forces were also reflected in M1 during mere movement observation. Specifically, it seemed that for all time points showing hand-object interactions (grasp, lift, place), a tight correspondence existed between observation-induced M1 facilitation and execution-induced muscle activity, whereas for other time points-showing no handobject interaction (i.e., at reach and return)-apparent deviations existed between M1 facilitation and executioninduced muscle activity (findings of deviant M1 facilitation at the early reach phase are discussed in more detail in the last paragraph).

Overall, data confirm previous evidence on an observation-to-execution matching mechanism within motor areas and extend these findings by showing that observation-induced changes in M1 excitability are time- and force-dependent.

Functional significance of time course-dependent force encoding in M1

A variety of proposals have been made regarding the purpose of observation-induced activity in motor areas (for 
review see Wilson \& Knoblich, 2005), suggesting that 'motor simulation' in the brain is used for facilitating overt imitation and observational learning (e.g., Iacoboni et al., 1999; Rizzolatti, Fogassi, \& Gallese, 2001), for understanding others' actions (de Lange et al., 2008; Gallese, Fadiga, Fogassi, \& Rizzolatti, 1996; Jeannerod, 2001), or to track the behavior of others in real time for generating perceptual predictions (i.e., the more recent 'emulation model' on action perception (Wilson \& Knoblich, 2005; Cattaneo et al., 2011). Although detailed descriptions of these models are beyond the scope of this paper, it should be noted that the various proposals are not mutually exclusive and that 'motor simulation' very likely plays a role in each of these cognitive activities (Wilson \& Knoblich, 2005)

Overall, data from the present study may best be interpreted and add support to the motor simulation account suggesting an activation of motor systems to emulate or understand the 'goal' or 'cause' of an action (de Lange, Spronk, Willems, Toni, \& Bekkering, 2008; Jeannerod, 2001). A previous study on weight perception tasks showed that observers relied most on the duration of the grasp and lifting movement to judge or infer weight and make less use of other cues, such as the duration of the place phase (Hamilton, Joyce, Flanagan, Frith, \& Wolpert, 2007). The present study also suggests that the most informative cues to infer object weight may have originated from the late grasp and lift phases, as the level of force encoding is most pronounced at these phases. Together, this and our study provide indications that accumulating kinematic cues on object weight during the grasp and lift phases are most salient to trigger a simulation process within motor areas and that this mechanism may be functionally relevant for inferring the weight of the lifted objects. In the present study, observers were asked to report the weight of the lifted object (after each block) and the overall recognition scores were considerably high (only 6 out of 16 subjects made occasional errors). However, due to this ceiling performance in weight recognition, it was not possible to explicitly test the relationship between correct weight reporting and excitability modulations in M1. It should, however, be interesting to test this in future studies (i.e., by adopting more challenging weight perception tasks).

Although the currently adopted TMS paradigm only allows the assessment of observation-induced changes in M1 excitability, other neurophysiological evidence exists on the role of the parieto-frontal mirror circuit in representing distinct features of observed actions. The parietal node, namely the inferior parietal lobule (IPL), was suggested to provide a 'goal description' of observed actions, by defining the identity and function of to-be-grasped objects, whereas the inferior frontal gyrus (IFG) is suggested to represent kinematic aspects of observed actions
(Grafton \& Hamilton, 2007; Hamilton \& Grafton, 2006; Pobric \& Hamilton, 2006; Tunik, Rice, Hamilton, \& Grafton, 2007). Although not formally part of the mirror network, it is suggested that action simulation in M1 may result from strong reciprocal connections to upstream fronto-parietal mirror regions. In this respect, M1 may be recruited to simulate muscle-related aspects of observed movements, such as the type of muscles and the muscular force requirements. The notion of a functional role of M1 in the process of mirror motor matching has been suggested before (Alaerts et al., 2009a, 2009b; Lepage, Lortie, \& Champoux, 2008; Kilner \& Frith, 2007; Pineda, 2008) and is supported by the identification of M1 neurons with mirror properties in the monkey's brain (Tkach, Reimer, \& Hatsopoulos, 2007). However, in keeping with findings by Avenanti, Bolognini, Maravita and Aglioti (2007)showing that interference with IFG also disrupted mirror motor activity in M1-the possibility cannot be ruled out that observation-induced M1 facilitation received crucial input from IFG mirror neurons.

\section{Early peak in force encoding at the reach phase}

Overall, the time course of force scaling in M1 during movement observation corresponded to the time course of force scaling during actual execution.

However, TMS data from the observation experiment consistently revealed an early peak in force-related M1 modulation already at the reach phase. This result was unexpected and no such force scaling was observed during actual execution. Subjects of the observation experiment were aware, however, that the same video clip was presented six times. Thus, after the first presentation, subjects had prior knowledge concerning the upcoming weight condition, which may have affected M1 excitability even during reaching, i.e., when no other visual cues about object weight were available (the to-be-lifted heavy/light objects were identical in appearance in all observation conditions). In the present active execution experiment, only peripheral electromyography recordings were performed at the muscle level, which may have been far too insensitive for unraveling similar dynamics at the early reach phase of execution. Using a more sensitive approach, however, a similar phenomenon has been reported for active action execution (Loh, Kirsch, Rothwell, Lemon, \& Davare, 2010). Loh et al. (2010) investigated the presence of a sensorimotor memory effect on corticomotor excitability while subjects actively lifted objects of different weights. In their study, M1 excitability was assessed at different times of the reaching phase and the size of motor evoked potentials was shown to be influenced by the previous lift, such that corticomotor excitability was larger when the action was preceded by lifting a heavy, as 
compared to a light, object. As such, these results clearly demonstrated that M1 excitability was scaled according to the weight of the object experienced in the previous lift when no external input concerning the object's weight was available (Loh et al., 2010). Apart from the study by Loh et al. (2010), several other studies also showed that M1 was part of a larger cortical network involved in storing a sensorimotor memory of the previous lift (Berner, Schonfeldt-Lecuona, \& Nowak, 2007; Chouinard, Leonard, \& Paus, 2005; Li, Padoa-Schioppa, \& Bizzi, 2001; Nowak, Voss, Huang, Wolpert, \& Rothwell, 2005). For example, when objects of different weights are lifted, grip force was shown to be scaled according to the previous lift such that the grip force was either too low when a lighter weight was expected or too high when a heavier weight was expected (Johansson \& Westling, 1988). Our results suggest that action observation can induce similar sensorimotor memory effects in M1, i.e., by modulating motor excitability in accordance with previously observed lifts. This is in line with a recent behavioral study showing that movement observation has a similar, albeit much weaker, effect on sensorimotor memory than movement execution when subjects grasp and lift a familiar object (Uçar \& Wenderoth, 2011).

Predictive scaling of force requirements already at the early reach phase may also support the notion that motor simulation during action perception is based on predictive coding. In essence, the predictive coding account asserts that action simulations are carried out to confirm prior expectations or predictions about action goals (de Haas, Greven, \& Kilner, 2011, de Lange, 2011; Kilner, Friston, \& Frith, 2007; Schutz-Bosbach \& Prinz, 2007; Stadler, Springer, Parkinson, \& Prinz, 2011; Zentgraf, Munzert, Bischoff, \& Newman-Norlund, 2011). More specifically, given a prior expectation about the goal/intention of an observed action, the motor system of the observer generates predictive representations (kinematics/motor commands) of other's actions, which subsequently are compared to the actually observed action (Kilner et al., 2007). The comparison of the predicted and actually observed action is essential in this process as the error between the two can be used to update the previously expected movement goal, i.e., by reciprocally minimizing the discrepancy between the predicted and observed kinematics/motor commands.

In line with the assumption that motor simulation is predictive, Umilta et al. (2001) showed that monkey mirror neurons also discharged when the final part of a grasping action could not be seen, but only inferred (Umilta et al., 2001). Similarly in humans, Kilner, Vargas, Duval, Blakemore and Sirigu (2004) demonstrated that the mere anticipation of a movement led to activity of the motor system in the observer. More specifically, when subjects expected to observe a movement of somebody else, a slow negative-going EEG potential (indicative of covert motor preparation) was measured before movement onset. The authors argued that this suggested an active role for the observer's motor system in making a prediction of another person's action, i.e., by generating a predictive motor representation ahead of movement initiation (Kilner et al., 2004). In a similar vein, Urgesi et al. (2010) showed that motor facilitation was maximal for snapshots of motion displaying ongoing but incomplete actions, indicating that the motor system was preferentially activated by the anticipatory or predictive simulation of upcoming action phases (Urgesi et al., 2010). Also, an eye tracking study by Flanagan and Johansson (2003)showed that during action observation, subjects implemented eye movement programs that were predictive of the upcoming movement, rather than being reactive (Flanagan \& Johansson, 2003). At a broader level, it has been suggested that the ability to predict and anticipate the future course of actions is relevant to achieve excellence in sports. As in, Aglioti, Cesari, Romani and Urgesi (2008) the investigation of action anticipation in basketball and the results indicated that only elite athletes were able to use finely tuned anticipatory 'resonance' mechanisms to predict others' actions ahead of their realization (Aglioti et al., 2008). Together, these findings indicate that action simulation not only mirrors the present state of an observed action, but also sets up an internal model in the observer to predict the forthcoming action ahead of its realization (Schutz-Bosbach \& Prinz, 2007). Our findings of an early peak in force-related M1 modulation already at the reach phase may suggest that the observer's motor system used force-related informationwhich was a priori available from the previously observed action (i.e., the same weight condition was shown repeatedly) - to predict the appropriate motor command of the upcoming, observed lifting action.

It is puzzling, however, as to why predictive force scaling was only apparent at the early reach phase and did not persist during the following grasping phase. Based on the present results alone, it is difficult to draw firm conclusions on this issue. However, Loh et al., (2010) proposed a model on the interaction between stored sensorimotor memory and available visual information. The results of this study indicated that a sensorimotor memory was formed based on the weight of previously lifted objects and that observers relied on this memory to prepare for upcoming lifts when no other visual cues were present. However as soon as visual cues (on the upcoming object weight) became available, this new information interacted with the stored representations and allowed the motor system to switch gradually to a state that corresponded to the newly available visual information (Loh et al., 2010). In our study, first kinematic cues on object 
weight only started from the beginning of grasping and accumulated until the late lifting phase. Although highly speculative, it can be hypothesized that the availability of this new visual information on object weight (which was, however, still very uncertain at the early grasping point) induced a switch such that M1 activity represented the observed movement characteristics rather than predictions based on the sensorimotor memory.

Nevertheless, more experiments are needed to replicate these findings using full-factorial designs (as in the present study, heavy weight conditions were always preceded by lifts of heavy objects and light weight conditions by lifts of light objects). Specifically, a more systematic exploration of observation-induced sensorimotor memory-using similar paradigms as those used in the execution experiment in-Loh et al. (2010) should be conducted to establish whether action observation can induce similar sensorimotor memory effects as action execution.

In conclusion, corticomotor excitability in M1 was shown to modulate according to the force requirements of observed grasping and lifting actions. Both during action observation and execution, force encoding gradually increased from the grasping until the late lifting phase. However, only during observation-not during executionan early peak in force encoding was revealed already during reaching. The findings may speak in favor of the motor simulation hypothesis, suggesting an activation of motor systems to predict or infer the weight of the lifted object.

Acknowledgments We are grateful to all the subjects who participated in this research and to Ellen Smets for her help in the data assessment process. Support for this study was provided through grants from the Flanders Fund for Scientific Research (FWO projects G.0292.05, G.0577.06 \& G.0758.10). KA was supported by an FWO postdoctoral research fellowship grant. This work was also supported by Grant P6/29 from the Interuniversity Attraction Poles program of the Belgian federal government.

\section{References}

Aglioti, S. M., Cesari, P., Romani, M., \& Urgesi, C. (2008). Action anticipation and motor resonance in elite basketball players. Nature Neuroscience, 11(9), 1109-1116.

Alaerts, K., Heremans, E., Swinnen, S. P., \& Wenderoth, N. (2009a). How are observed actions mapped to the observer's motor system? Influence of posture and perspective. Neuropsychologia, $47(2), 415-422$

Alaerts, K., Senot, P., Swinnen, S. P., Craighero, L., Wenderoth, N., \& Fadiga, L. (2010a). Force requirements of observed object lifting are encoded by the observer's motor system: a TMS study. European Journal of Neuroscience, 31(6), 1144-1153.

Alaerts, K., Swinnen, S. P., \& Wenderoth, N. (2009b). Is the human primary motor cortex activated by muscular or directiondependent features of observed movements? Cortex, 45(10), $1148-1155$.
Alaerts, K., Swinnen, S. P., \& Wenderoth, N. (2010b). Observing how others lift light or heavy objects: which visual cues mediate the encoding of muscular force in the primary motor cortex? Neuropsychologia, 48(7), 2082-2090.

Avenanti, A., Bolognini, N., Maravita, A., \& Aglioti, S. M. (2007). Somatic and motor components of action simulation. Current Biology, 17(24), 2129-2135.

Berner, J., Schonfeldt-Lecuona, C., \& Nowak, D. A. (2007). Sensorimotor memory for fingertip forces during object lifting: The role of the primary motor cortex. Neuropsychologia, 45(8), 1931-1938.

Borroni, P., Montagna, M., Cerri, G., \& Baldissera, F. (2005). Cyclic time course of motor excitability modulation during the observation of a cyclic hand movement. Brain Research, 1065(1-2), $115-124$.

Buccino, G., Binkofski, F., Fink, G. R., Fadiga, L., Fogassi, L., Gallese, V., et al. (2001). Action observation activates premotor and parietal areas in a somatotopic manner: An fMRI study. European Journal of Neuroscience, 13(2), 400-404.

Cattaneo, L., Barchiesi, G., Tabarelli, D., Arfeller, C., Sato, M., \& Glenberg, A. M. (2011). One's motor performance predictably modulates the understanding of others' actions through adaptation of premotor visuo-motor neurons. Social Cognitive and Affective Neuroscience, 6(3), 301-310.

Chouinard, P. A., Leonard, G., \& Paus, T. (2005). Role of the primary motor and dorsal premotor cortices in the anticipation of forces during object lifting. Journal of Neuroscience, 25(9), $2277-2284$

de Haas, Greven and Kilner (2011) Why the motor system is active when observing other people's actions. Special Issue Psychological Research.

de Lange, F. P., Spronk, M., Willems, R. M., Toni, I., \& Bekkering, H. (2008). Complementary systems for understanding action intentions. Current Biology, 18(6), 454-457.

deLange (2011) Why and what do we simulate during the perception of actions?Special Issue Psychological Research.

Devanne, H., Lavoie, B. A., \& Capaday, C. (1997). Input-output properties and gain changes in the human corticospinal pathway. Experimental Brain Research, 114(2), 329-338.

Di Pellegrino, G., Fadiga, L., Fogassi, L., Gallese, V., \& Rizzolatti, G. (1992). Understanding motor events: a neurophysiological study. Experimental Brain Research, 91(1), 176-180.

Fadiga, L., Fogassi, L., Pavesi, G., \& Rizzolatti, G. (1995). Motor facilitation during action observation: a magnetic stimulation study. Journal of Neurophysiology, 73(6), 2608-2611.

Flanagan, J. R., \& Johansson, R. S. (2003). Action plans used in action observation. Nature, 424(6950), 769-771.

Fogassi, L., Ferrari, P. F., Gesierich, B., Rozzi, S., Chersi, F., \& Rizzolatti, G. (2005). Parietal lobe: From action organization to intention understanding. Science, 308(5722), 662-667.

Gallese, V. (2009). Mirror neurons, embodied simulation, and the neural basis of social identification. Psychoanalytic Dialogues, 19(5), 519-536.

Gallese, V., Fadiga, L., Fogassi, L., \& Rizzolatti, G. (1996). Action recognition in the premotor cortex. Brain, 119(2), 593-609.

Gallese, V., Keysers, C., \& Rizzolatti, G. (2004). A unifying view of the basis of social cognition. Trends in Cognitive Sciences, 8(9), 396-403.

Gangitano, M., Mottaghy, F. M., \& Pascual-Leone, A. (2001). Phasespecific modulation of cortical motor output during movement observation. Neuroreport, 12(7), 1489-1492.

Grafton, S. T., Arbib, M. A., Fadiga, L., \& Rizzolatti, G. (1996). Localization of grasp representations in humans by positron emission tomography.2. Observation compared with imagination. Experimental Brain Research, 112(1), 103-111. 
Grafton, S. T., \& Hamilton, A. F. (2007). Evidence for a distributed hierarchy of action representation in the brain. Human Movement Science, 26(4), 590-616.

Grezes, J., Armony, J. L., Rowe, J., \& Passingham, R. E. (2003). Activations related to "mirror" and "canonical" neurones in the human brain: An fMRI study. Neuroimage, 18(4), 928-937.

Hamilton, A., \& Grafton, S. T. (2006). Goal representation in human anterior intraparietal sulcus. Journal of Neuroscience, 26(4), $1133-1137$.

Hamilton, A. F. D., Joyce, D. W., Flanagan, J. R., Frith, C. D., \& Wolpert, D. M. (2007). Kinematic cues in perceptual weight judgement and their origins in box lifting. Psychological Research, 71(1), 13-21.

Hess, C. W., Mills, K. R., \& Murray, N. M. (1987). Responses in small hand muscles from magnetic stimulation of the human brain. Journal of Physiology, 388, 397-419.

Iacoboni, M., Woods, R. P., Brass, M., Bekkering, H., Mazziotta, J. C., \& Rizzolatti, G. (1999). Cortical mechanisms of human imitation. Science, 286(5449), 2526-2528.

Jeannerod, M. (2001). Neural simulation of action: A unifying mechanism for motor cognition. Neuroimage, $14((1 \mathrm{Pt} 2))$, S103-S109.

Johansson, R. S., \& Westling, G. (1988). Coordinated isometric muscle commands adequately and erroneously programmed for the weight during lifting task with precision grip. Experimental Brain Research, 71(1), 59-71.

Kilner, J. M., Friston, K. J., \& Frith, C. D. (2007). The mirror-neuron system: A Bayesian perspective. Neuroreport, 18(6), 619-623.

Kilner, J. M., \& Frith, C. D. (2007). A possible role for primary motor cortex during action observation. Proceedings of the National Academy of Sciences USA, 104(21), 8683-8684.

Kilner, J. M., Vargas, C., Duval, S., Blakemore, S. J., \& Sirigu, A. (2004). Motor activation prior to observation of a predicted movement. Nature Neuroscience, 7(12), 1299-1301.

Lepage, J. F., Lortie, M., \& Champoux, F. (2008). Action-coding neurons in primary motor cortex: Making sense of M1 activity during action perception. Journal of Neuroscience, 28(9), 1995-1996.

Li, C. S. R., Padoa-Schioppa, C., \& Bizzi, E. (2001). Neuronal correlates of motor performance and motor learning in the primary motor cortex of monkeys adapting to an external force field. Neuron, 30(2), 593-607.

Loh, M. N., Kirsch, L., Rothwell, J. C., Lemon, R. N., \& Davare, M. (2010). Information about the weight of grasped objects from vision and internal models interacts within the primary motor cortex. Journal of Neuroscience, 30(20), 6984-6990.

Maeda, F., Kleiner-Fisman, G., \& Pascual-Leone, A. (2002). Motor facilitation while observing hand actions: Specificity of the effect and role of observer's orientation. Journal of Neurophysiology, 87(3), 1329-1335.

Montagna, M., Cerri, G., Borroni, P., \& Baldissera, F. (2005). Excitability changes in human corticospinal projections to muscles moving hand and fingers while viewing a reaching and grasping action. European Journal of Neuroscience, 22(6), $1513-1520$.

Nowak, D. A., Voss, M., Huang, Y. Z., Wolpert, D. M., \& Rothwell, J. C. (2005). High-frequency repetitive transcranial magnetic stimulation over the hand area of the primary motor cortex disturbs predictive grip force scaling. European Journal of Neuroscience, 22(9), 2392-2396.

Oldfield, R. C. (1971). The assessment and analysis of handedness: the Edinburgh Inventory. Neuropsychologia, 9(1), 97-113.

Pineda, J. A. (2008). Sensorimotor cortex as a critical component of an 'extended' mirror neuron system: Does it solve the development, correspondence, and control problems in mirroring? Behavioral Brain Function, 4, 47.

Pobric, G., \& Hamilton, A. F. D. (2006). Action understanding requires the left inferior frontal cortex. Current Biology, 16(5), 524-529.

Rizzolatti, G., Fadiga, L., Gallese, V., \& Fogassi, L. (1996). Premotor cortex and the recognition of motor actions. Cognitive Brain Research, 3(2), 131-141.

Rizzolatti, G., Fogassi, L., \& Gallese, V. (2001). Neurophysiological mechanisms underlying the understanding and imitation of action. Nature Reviews Neuroscience, 2(9), 661-670.

Rizzolatti, G., \& Sinigaglia, C. (2010). The functional role of the parieto-frontal mirror circuit: Interpretations and misinterpretations. Nature Reviews Neuroscience, 11(4), 264-274.

Romani, M., Cesari, P., Urgesi, C., Facchini, S., \& Aglioti, S. M. (2005). Motor facilitation of the human cortico-spinal system during observation of bio-mechanically impossible movements. Neuroimage, 26(3), 755-763.

Rossini, P. M., Barker, A. T., Berardelli, A., Caramia, M. D., Caruso, G., Cracco, R. Q., et al. (1994). Noninvasive electrical and magnetic stimulation of the brain, spinal-cord and roots: Basic principles and procedures for routine clinical-application. Report of an IFCN committee. Electroencephalography and Clinical Neurophysiology, 91(2), 79-92.

Schutz-Bosbach, S., \& Prinz, W. (2007). Prospective coding in event representation. Cognitive Processing, 8(2), 93-102.

Sinigaglia, C., \& Sparaci, L. (2010). Emotions in action through the looking glass. Journal of Analytical Psychology, 55(1), 3-29.

Stadler, Springer, Parkinson, Prinz (2011). Self-other commensurability of movement dynamics affects action prediction. Special Issue Psychological Research.

Strafella, A. P., \& Paus, T. (2000). Modulation of cortical excitability during action observation: A transcranial magnetic stimulation study. Neuroreport, 11(10), 2289-2292.

Tkach, D., Reimer, J., \& Hatsopoulos, N. G. (2007). Congruent activity during action and action observation in motor cortex. Journal of Neuroscience, 27(48), 13241-13250.

Tunik, E., Rice, N. J., Hamilton, A., \& Grafton, S. T. (2007). Beyond grasping: Representation of action in human anterior intraparietal sulcus. Neuroimage, 36(2), 77-86.

Uçar, E., \& Wenderoth, N. (2011). Movement observation affects sensorimotor memory when lifting a familiar object. Cortex. doi: 10.1016/j.cortex.2011.08.011.

Umilta, M. A., Kohler, E., Gallese, V., Fogassi, L., Fadiga, L., Keysers, C., et al. (2001). I know what you are doing: A neurophysiological study. Neuron, 31(1), 155-165.

Urgesi, C., Candidi, M., Fabbro, F., Romani, M., \& Aglioti, S. M. (2006). Motor facilitation during action observation: Topographic mapping of the target muscle and influence of the onlooker's posture. European Journal of Neuroscience, 23(9), 2522-2530.

Urgesi, C., Maieron, M., Avenanti, A., Tidoni, E., Fabbro, F., \& Aglioti, S. M. (2010). Simulating the future of actions in the human corticospinal system. Cerebral Cortex, 20(11), 2511-2521.

Wilson, M., \& Knoblich, G. (2005). The case for motor involvement in perceiving conspecifics. Psychological Bulletin, 31(3), 460-473.

Zentgraf, K., Munzert, J., Bischoff, M., \& Newman-Norlund, R. D. (2011). Simulation during observation of human actions- theories, empirical studies, applications. Vision Research, 51(8), $827-835$. 